\title{
CONSTRUCCIÓN DE PAZ Y CIUDADANÍA. ENTRE LA INDIFERENCIA Y LA MEMORIA
}

Peace building and citizenship. Between indifference and memory

\section{Artículo de reflexión derivado de investigación}

DOI: https://doi.org/10.21501/23461780.3843

Recibido: 7 de mayo de 2020 / Aceptado: 30 de noviembre de 2020 / Publicado: 14 de diciembre de 2020

\section{Diego León Cossio Sepúlveda*, Alexander Hincapié García}

\section{Resumen}

Este artículo destaca el papel de la memoria en la formación ciudadana y la construcción de paz para señalar su carácter formador. Su objetivo es demostrar que la indiferencia, como producto de la ausencia de memoria, es una forma de violencia posible por condiciones históricas, culturales, simbólicas y materiales que configuran una subjetividad indiferente. En primer lugar, se realiza un acercamiento a los conceptos de violencia y conflicto para argumentar por qué la indiferencia es un tipo específico de violencia. En un segundo momento, se argumenta que la memoria edifica virtudes como la responsabilidad y la

\footnotetext{
a El presente artículo surge del trabajo de investigación doctoral titulado Formación ciudadana y memoria. Una interpretación desde la teoría del conocimiento de Manuel Reyes Mate.

* Magíster en Gobierno y Políticas Públicas, estudiante de Doctorado en Ciencias de la Educación, Universidad de San Buenaventura, Medellín, Colombia, participante del Grupo Interdisciplinario de Estudios Pedagógicos (GIDEP), ORCID: https://orcid.org/0000-0001-8428-2188. Contacto: dlcossio@gmail.com

** Doctor en Educación, docente titular de la Universidad de San Buenaventura, Medellín, Colombia, participante del Grupo Interdisciplinario de Estudios Pedagógicos (GIDEP), ORCID: https://orcid.org/0000-0001-6828

2786. Contacto: alexander.hincapie@usbmed.edu.co.
} 
tolerancia, llamadas a ser la base de la formación de una ciudadanía para la paz. Finalmente, se establece el vínculo entre memoria y justicia para sostener que sin justicia no hay paz.

\title{
Palabras clave
}

Construcción de paz; Ciudadanía; Indiferencia; Violencia; Responsabilidad; Tolerancia.

\begin{abstract}
This article highlights the role of memory in civic education and in peace building, emphasizes its formative character. Its objective is to demonstrate that indifference, as a result of lack of memory, is a form of violence; feasible due to historical, cultural, symbolic and material conditions shaping an indifferent subjectivity. Thus, in the first place an approach is made to the concepts of violence and conflict to argue why the indifference is a specific type of violence. In a second instance, it is argued how memory builds virtues such as responsibility and tolerance, called to be the basis for citizenship education for peace. Finally, the link between memory and justice is established, to argue that there is no peace without justice.
\end{abstract}

\section{Keywords}

Peace building; Citizenship; Indifference; Violence; Responsibility; Tolerance. 


\section{Introducción}

La obra de Reyes Mate ofrece un campo fecundo para el estudio y análisis de cuestiones como el progreso y la ciudadanía. Su reflexión en torno a Auschwitz - paradigma de lo atroz-, sigue la línea argumentativa de pensadores como Benjamin y Adorno, por lo que supone una mirada diferente sobre el problema de la violencia. Desde la teoría del conocimiento - teoría de la memoriaexpuesta por Reyes Mate, este artículo busca mostrar cómo concebir la indiferencia como una forma de violencia. Por lo tanto, la pregunta por las condiciones que generan la indiferencia demanda comprender la relación entre el hombre y las condiciones materiales que lo determinan, así como la orientación de la formación ciudadana.

En un primer momento, en el artículo se parte de la concepción de violencia de Johan Galtung para evidenciar que la indiferencia es el fenómeno que permite la reproducción de las violencias estructural y cultural, así, la indiferencia se eleva a una forma específica de violencia. Es el producto de una razón instrumental que normaliza las formas en las que la violencia se reproduce.

En un segundo momento, se establece la relación entre la formación ciudadana y la construcción de paz, y con ello, la necesidad de repensar la ciudadanía a partir de las ideas de Manuel Reyes Mate. Aquí se propone que la reconstrucción anamnética de virtudes como la responsabilidad y la tolerancia ocupa un lugar central en la generación de justicia. La recuperación del pasado permite poner de manifiesto la forma como se han constituido las condiciones materiales que permiten la violencia por indiferencia.

Finalmente, en un tercer momento, el artículo se ocupa de mostrar que la justicia es un elemento determinante en la construcción de paz; la justicia requiere de la memoria para concretarse, toda vez que la falta de memoria produce sociedades incapaces de cuestionar la base de lo que ha llegado a ser, es decir, la injusticia y las condiciones que producen la violencia. 


\section{Método}

El enfoque previsto para la construcción del artículo se encuentra dentro de la perspectiva de la filosofía de la educación. Siguiendo de manera heterodoxa a Fullat (1987), esta perspectiva se entiende como un saber comprensivo y crítico que permite identificar los presupuestos antropológicos de la formación, analizar sus límites e interrogar su necesidad. En términos generales, el método utilizado es filosófico y consiste en el planteamiento, exposición, cuestionamiento y reconfiguración de un argumento. Como sugiere Piñeres Sus (2017), el método es inseparable de procedimientos como la selección de las fuentes, las relaciones construidas entre ellas y las apropiaciones consumadas en función de un argumento particular; pero, más allá de todo esto, el método es indisociable de la actividad escritural. Si es imperativo declarar lo más importante de la construcción metodológica, lo justo será decir que el método está en el esfuerzo por depurar la escritura y, a través de esto, arrancar los problemas planteados al sentido común que gobierna la interpretación de las cosas. De manera conceta, el trabajo parte de la revisión documental de la obra de Reyes Mate, en la que la memoria o razón del sufrimiento es piedra basal del pensamiento, para dar paso a un análisis categorial donde construcción paz, ciudadanía e indiferencia se iluminan a la luz de la razón de los vencidos, esto es, de la memoria de las víctimas, sin las cuales no es posible ampliar el marco interpretativo de la justicia.

Tabla 1. Método de investigación

\begin{tabular}{|c|c|c|}
\hline \multicolumn{3}{|c|}{ Enfoque a partir de la Filosofia de la educación } \\
\hline $\begin{array}{l}\text { Exposición y planteamiento del } \\
\text { problema en términos de: }\end{array}$ & $\begin{array}{l}\text { Cuestionamiento a la luz de la teoría del } \\
\text { conocimiento de Reyes Mate }\end{array}$ & Reconfiguración del argumento \\
\hline $\begin{array}{l}\text { - Construcción de paz } \\
\text { - Ciudadanía } \\
\text { - Indiferencia }\end{array}$ & $\begin{array}{l}\text { La memoria como criterio específico de } \\
\text { indagación e interrogación }\end{array}$ & $\begin{array}{l}\text { Nuevas categorías de análisis: } \\
\text { estructura alcanzada }\end{array}$ \\
\hline Criterio metodológico constante del & ulo: atención a la escritura como for & ando \\
\hline
\end{tabular}




\section{Conflicto y violencia como resultado de la indiferencia}

Todo planteamiento sobre la educación para la paz debe expresar de forma clara cómo se entiende el conflicto y la violencia; comprender ambos fenómenos permite plantearse un proceso educativo que verdaderamente pueda asumir el imperativo de la paz. Asimismo, es importante destacar que conflicto y violencia, como conceptos, tienen características propias; si bien están relacionados, no son equiparables. Por ello, "la paz es lo que tenemos cuando sucede de forma pacífica la transformación creativa del conflicto" (Galtung, 1995, p. 236); sin desconocer que no todo conflicto desencadena necesariamente violencia. De esta manera se pueden deducir, al menos, tres puntos: toda violencia es resultado de un conflicto; no todo conflicto deriva en violencia; $y$ la violencia no es el origen del conflicto.

Debido a que toda violencia es producto de un conflicto, conviene precisar que la existencia de conflictos es inherente a las sociedades; en primer lugar, porque estos son producto de las relaciones de poder establecidas entre los hombres; en segundo lugar, porque la aspiración y el derecho a la libertad de sus miembros es fuente de diferencias. Como señalan Köhle (1974) y Zdarzil (1978), la ausencia de conflictos solo es posible eliminando las relaciones de poder o suprimiendo las libertades individuales - aspecto impensable dentro de las sociedades modernas-. Así, "en el interior profundo de cada conflicto hay una contradicción, algo que se interpone en el camino de otra cosa" (Galtung, 2003, p. 107) que al no encontrar un mecanismo de solución acude a la violencia para imponer una visión unilateral. Por esta razón, la solución no violenta de los conflictos depende de la capacidad de las partes involucradas para encontrar soluciones creativas; pero, además, depende de una formación ciudadana que ayude a los individuos a reconocer a los otros como sujetos sociales y políticos.

La concepción tradicional de la violencia hace referencia al uso de la imposición de la fuerza como medio para resolver los conflictos, y recibe el nombre de violencia directa para diferenciarla de las violencias estructural y cultural (Galtung, 2003). De acuerdo con Galtung, la violencia directa es aquella que se hace visible mediante la agresión física; la violencia estructural se carac- 
teriza por la privación de derechos fundamentales, con el establecimiento en sociedad de instituciones y prácticas que niegan los derechos y, finalmente, la violencia cultural hace alusión a los imaginarios y valores existentes en la sociedad, o en una parte de ella, para justificar la supresión de los derechos 0 legitimar el uso de la violencia directa. Es específico de la violencia cultural la producción de representaciones degradantes en torno a determinados grupos sociales, por ejemplo, sobre los judíos durante el régimen nazi o sobre los homosexuales en contextos homofóbicos. En la línea argumentativa de Erving Goffman (2006), podría decirse que esas representaciones son estigmas que tienen por propósito inocular el desprecio y la repulsión en la subjetividad de los estigmatizados.

Entre la violencia estructural y la violencia cultural —violencias de cierta forma invisibles si se las compara con la violencia directa - tiene lugar un fenómeno social que permite la reproducción de ambas formas de violencia: la indiferencia. Esta puede concebirse como otra forma de violencia caracterizada por la no acción frente a las condiciones históricas, culturales, simbólicas y materiales que producen la violencia, y la justificación o minimización de la injusticia. La indiferencia se presenta como racional por su sintonía con las tendencias sociales de la época; una forma aberrante que Hegel (2004) capta muy bien al declarar que lo real es racional y lo racional es real. Es decir, no hay contradicción moral en aceptar el daño causado a los otros, si, por ejemplo, a cambio, ese daño se justifica con la promesa del progreso para la mayoría. Así, la indiferencia es un tipo de práctica social considerada correcta, por lo tanto, racional, que contribuye a reproducir la violencia y las condiciones de injusticia.

La indiferencia es más que el desconocimiento de las violencias estructural y cultural, es el resultado de una razón instrumental que concibe como normal o inevitable el daño que se causa o padecen determinados grupos sociales. No es difícil pensar que la indiferencia sea incluso un valor entre quienes se representan como sujetos conscientes y críticos; esto significa que la indiferencia no puede ser superada solo con el desarrollo de la consciencia. Tiene que superarse con la transformación de las condiciones materiales que se justifican en la consciencia indiferente, toda vez que, como señala Hincapié García 
(2016a), las condiciones subjetivas no pocas veces son cómplices, entre otras cosas, porque "el hombre no está más allá de las condiciones que él mismo ha creado en el proceso de formarse" (Hincapié García, 2016a, p. 261), a las cuales se les ha atribuido un carácter inevitable que diezma el espíritu de cambio. La transformación exige un costo que las sociedades no están dispuestas a pagar, pues ello implica arriesgar lo que ya se posee 0 enfrentar la incertidumbre de luchar contra lo que parece inevitable.

En línea con lo anterior, la indiferencia se pliega a las dimensiones del poder vigente, pero también de la subjetividad social asignada. Es el producto de la incapacidad de autorreflexión, juicio y reacción de los individuos (Adorno, 1998) frente a situaciones no solo de violencia, sino de conflicto. En otras palabras, la indiferencia hace posible que los actos de violencia directa, estructural y cultural no sean vistos con preocupación, ni se requiera interrogar las condiciones que propician la violencia. Aquí cabe recordar a Walter Benjamin (2010) afirmando que la educación hasta ahora recibida trabaja por convertir a los seres humanos en indiferentes. Entre otras cosas, porque no se educa para que los seres humanos puedan ir a la raíz -injusta — de su formación, sino para aceptar como realidad aquellos derechos formales que no pueden realizarse como la igualdad y la libertad.

Si la superación de la violencia depende de la superación de la indiferencia, es necesario comprender cómo se llegó a esta última. De acuerdo con Reyes Mate $(2003,2008 a, 2009)$, la indiferencia refleja la ausencia de memoria y debe estudiarse como el resultado del proyecto moderno que emergió de las ideas ilustradas: superación de la tradición, rechazo de los elementos no racionales, dominio de la naturaleza y orientación hacia un futuro que se representa prometedor. En esa línea, la memoria es "el principio del pensar" (Mate, 2011, p. 173), dado que se constituye como la capacidad de cuestionar el presente a partir de la visión del vencido, permitiendo diferenciar realidad de facticidad. Para el filósofo español, "lo que no ha llegado a ser y ha quedado arrumbado en las cunetas de la historia es parte de la realidad, aunque no lo sea de la facticidad" (Mate, 2013. p. 59). En otras palabras, la realidad contiene la posibilidad y la facticidad, lo dado. En este contexto, la razón es reducida a la racionalidad científica y privada de sus fuerzas históricas y sociales. Con ello se "convierte a 
la razón en un peligroso instrumento capaz de llevar a cabo las más insensatas empresas" (Mate, 2008a, p. 8), pues se muestra indiferente a las consecuencias que puede traer la racionalidad científica sobre la sociedad.

La ausencia de memoria produce sociedades incapaces de cuestionar los hechos que están en la base de lo que ha llegado a ser la sociedad. La ausencia de memoria produce una parálisis reflexiva y crítica que imposibilita identificar las condiciones que dividen la sociedad en vencedores y vencidos, así como las causas que hacen posible el imperio de la violencia. La memoria en una sociedad debe conducir a la superación de la indiferencia y a la asunción de la responsabilidad de toda la sociedad por cada acto de violencia que ha tenido y tiene lugar, pues las causas de la violencia radican en las condiciones que han sido configuradas por la acción del ser humano en sociedad, de manera que su transformación depende de recuperar el pasado para evidenciar los presentes negados y permitir la posibilidad de la transformación de la sociedad.

El proyecto moderno, y la lógica de progreso que se impone como fin de las sociedades occidentales, establece una dialéctica demoníaca entre vencedores y vencidos. Los vencidos son el costo para alcanzar el bienestar que el progreso promete (Mate, 2009). De ahí que la idea de progreso no puede concebirse sin violencia. Hay otro progreso que tiene que ver con la justicia, sugiere Benjamin (2012), el cual solo es posible si se parte de la memoria. Ahora bien, en el momento en que la modernidad disuelve el reino de la moralidad en el ámbito de las leyes, ese otro progreso no es posible, es bloqueado por la versión ruinosa que impera manifiestamente en la dialéctica entre vencedores y vencidos.

Dicho sin ambivalencias, la violencia es resultado de las condiciones históricas, culturales, simbólicas y materiales o de lo que Adorno llama "el orden social que produce y reproduce la frialdad" (Adorno, 1998, p. 90). Estas no solo posibilitan el estado de cosas que legitima las tres formas de violencia señaladas por Galtung, sino que también condicionan o influencian la actuación del hombre, incluso cuando este pueda decir de sí mismo que es un sujeto consciente que rechaza las causas de la violencia. Expuesto así, como ya se indicó, la transformación de la sociedad "no puede realizarse meramente en la concien- 
cia" (Hincapié García, 2016b, p. 55). En sentido estricto, las condiciones históricas, culturales, simbólicas y materiales son las que producen la modalidad de la consciencia, de lo que es posible deducirse que, en parte, la consciencia es solo su reflejo. Esto implica que el desarrollo de la consciencia crítica no es suficiente, como argumenta Jiménez Bautista (2012), "la violencia del ser humano no está en sus genes sino en su ambiente" (p. 14) y, por lo tanto, la educación para la paz no solo debe formar en contra de la violencia, sino en contra del ambiente que la genera. Apropiando este uso terminológico, ambiente aquí significa la sociedad petrificada en prácticas de dominación y explotación.

La educación para la paz tiene el reto de repensar la educación más allá de la razón instrumental. En efecto, puede apelar a una dialéctica de la Ilustración que confíe en la razón como elemento emancipador, sin desconocer que, a lo largo de la historia, la razón no ha estado exenta de tropiezos (Mate, 2008a). La educación para la paz debe avivar, a través de la memoria, los motivos que impulsen tanto la transformación de la subjetividad, como de las condiciones materiales que propician la violencia. El acto educativo debe ocuparse de la indiferencia, pues esta no solo es el vaso comunicante entre los distintos tipos de violencia, sino que en sí misma es otra forma de violencia. Como señala Adorno, la indiferencia mutila la capacidad del yo para verse en el no yo y, por lo tanto, no hay oposición a la violencia, a veces, exterminadora, que puede ejercerse en contra de los otros.

\section{La formación ciudadana y la educación para la paz}

La indiferencia como una forma de violencia debe ser abordada tanto desde la educación para la paz como desde la formación ciudadana; ambas tienen como finalidad asegurar la convivencia social. No obstante, es necesario resaltar que, aunque la construcción de paz se enmarque dentro de la formación política (Zdarzil, 1978), esta no se debe limitar a la búsqueda de soluciones no violentas a los conflictos que se presentan en la sociedad, sino a la creación de condiciones tanto diferentes como nuevas que posibiliten la paz. 
Concebir la construcción de paz a partir de la formación ciudadana y vincular esta última a virtudes que promueven la convivencia, busca identificar los puntos de convergencia entre ambas. Para Reyes Mate las virtudes que deben guiar la práctica ciudadana son la responsabilidad y la tolerancia, dado que ambas no solo permiten la construcción de la vida social, sino que, además, demandan en el ciudadano un compromiso con todos los miembros de la sociedad (Mate, 2016). El compromiso no se agota en el respeto a la diferencia o la inclusión de las minorías, sino que demanda acoger las luchas sociales como una responsabilidad común que es la base de la construcción de paz.

En este sentido, la construcción de paz es la expresión de una formación ciudadana que ha logrado hacer responsables a los seres humanos de la solución no violenta de los conflictos. Para Adorno, por ejemplo, la cuestión es que el hombre comprenda su papel histórico, pues no se trata de que sea un mero espectador (Adorno, 1998) o peor, un reproductor de los mecanismos que incentivan la violencia; se trata de la apertura del hombre hacia el mundo, encontrándose en cada una de sus partes, es decir, la conciencia sobre la realidad y las condiciones materiales que actúan en la formación del hombre. En ese sentido, la responsabilidad como virtud de la ciudadanía busca hacer evidente que "las consecuencias de la acción afectan al presente y al futuro" (Mate, 2016, p. 47), razón por la cual esta no es un asunto exclusivo de un grupo particular, sino de todos los ciudadanos en tanto los beneficios no son privados, sino colectivos. La indiferencia no solo tiene repercusiones sobre el presente, sino también lo hace sobre el futuro; legitimar las condiciones que dan lugar a la violencia contribuye a su reproducción. Otra vez, como sostiene Benjamin (2020), los vencidos de hoy heredan su situación de los vencidos ayer y, en consecuencia, legitimar la violencia en el presente contribuye a legitimarla en un tiempo después, de modo que la ruptura de esta forma de violencia requiere de la responsabilidad como virtud ciudadana para hacerse cargo de la injusticia.

La responsabilidad implica develar la indiferencia con la que se ha constituido el presente. El hombre debe hacerse responsable de los daños causados ayer y que se prolongan hoy. Con lo cual se busca mirar el presente, no desde los ojos del vencedor, sino desde la mirada de los vencidos, para transformarlo. La teoría del conocimiento que se encuentra en la obra de Reyes Mate ve en la 
visión del vencido las posibilidades que la facticidad del vencedor ha dado por canceladas. Por eso, la formación ciudadana desde la pespectiva de la memoria se refiere al hombre que interroga el presente y asume su tiempo a partir del sufrimiento del pasado. La indiferencia es contraria a la ciudadanía como expresión de convivencia social, debido a que se trata "de hacerse responsable de la vida misma, de la perpetuación indefinida de la humanidad. Puesto que el hombre tiene la posibilidad de destruir a la humanidad, tiene entonces la obligación de evitarlo" (Mate, 2016, p. 48).

El punto de partida de la responsabilidad, en este contexto, es la memoria. Al ciudadano le bastaría con ver cómo se ha establecido el presente y cuánto clama el pasado para reconocer su propia vulnerabilidad. Al interrogar el presente, optaría por afrontar la responsabilidad de transformar las condiciones que legitiman o promueven la violencia, para contribuir a la construcción de paz y a la formación de una ciudadanía responsable de edificar la vida en común. La memoria no solo busca una reparación material de los daños producidos por la violencia, sino también la recuperación de la responsabilidad como virtud de la ciudadanía (Mate, 2016), en tanto los males sociales no son causados por una persona concreta, sino por la complicidad de la sociedad (Arteta, 2010). Una sociedad que no procura una formación ciudadana fundamentada en la responsabilidad como virtud ciudadana no podrá construir la paz que demanda.

El ciudadano responsable reconoce que, por encima de la racionalidad instalada socialmente, reproductora de la injusticia, existe una verdad moral que reclama cambio, "el nuevo sujeto moral es un sujeto responsable, alguien que finalmente se hace responsable de los problemas de su tiempo y busca una solución" (Mate, 2016, p. 52). La responsabilidad tiene un doble carácter para el ejercicio de la ciudadanía: por un lado, implica asumirse como sujeto histórico que debe procurar el cambio de las condiciones que determinan su existencia; por otro lado, es una responsabilidad con quienes en el pasado padecieron la violencia. No asumir este compromiso solo puede traer como resultado la negación de una realidad que no solo genera violencia, sino que se ha construido sobre el olvido y es allí precisamente donde cobra importancia la memoria. El nuevo sujeto moral reconoce que, por malo que sea el mundo, él ha nacido para repararlo (Tikun). 
Asimismo, la construcción de paz demanda en el ciudadano pensar en los otros con los cuales se habita, debido a que "la pregunta del otro es la que permite verse a uno mismo implicado en esa responsabilidad. Eso quiere decir que uno no descubre su responsabilidad hurgando en su conciencia, sino escuchando una pregunta que viene de fuera" (Mate, 1998, p. 53). En otras palabras, no es posible construir paz cuando se es indiferente a lo que sucede con aquellos con los cuales se comparte en la sociedad. La indiferencia no solo agudiza las situaciones que avivan conflictos irresolubles, sino que alimenta el resentimiento que desencadena la violencia. La tradición judía enseña que en el amor al prójimo reposa la capacidad del hombre de humanizarse, en la respuesta al llamado del otro se reafirma o se cuestiona la humanidad, toda vez que, el otro, en su sufrimiento, interpela la respuesta del yo. Para Cohen (2004), el extranjero, ese otro frente al cual el yo se siente más distante, es el máximo elemento creador, profunda fuente de reflexiones, de donde surgen las ideas morales del judaísmo: "la idea de que Dios ama al forastero conecta la idea, con la que empieza la vocación de Israel, con aquella otra idea, con la que llega a su conclusión la vocación de Israel, a saber, con la idea de la unidad mesiánica del ser humano" (Cohen, 2004, p. 34). Esto indica que la responsabilidad que obliga a corregir la indiferencia no solo cobija a los más próximos, sino que, incluso, nos hace responsables por aquellos que consideramos los más ajenos a nuestro yo.

Al lado de la responsabilidad en el proceso de la construcción de paz, también se hace necesario formar una ciudadanía tolerante, debido a que, en primer lugar, la existencia inevitable de conflictos puede desencadenar en acciones violentas cuando no se tolera la diferencia; en segundo lugar, las sociedades democráticas demandan el ejercicio de la libertad de los ciudadanos para la formación de su subjetividad. Siguiendo a Piñeres Sus (2016), se puede agregar que la intolerancia, lo opuesto a la tolerancia, esconde formas de idealidad y, por lo tanto, de violencia. La paz de los vencedores es una forma de idealidad que se sustenta sobre la imposición de un determinado tipo de valores y de sociedad, que niega otras posibilidades y oculta aquellas que han resultado vencidas. Si la idealidad tiene lugar es porque la indiferencia la hace posible, pues con ella se afirma la negación de lo que no se representa como ideal. 
La tolerancia en la obra de Reyes Mate no consiste en "que se suspende la aplicación de una norma para permitir algo a alguien sin aprobarlo expresamente" (Mate, 2016, p. 64), es decir, en respetar algo con lo que no se comparte solo para evitar el conflicto, pues como se señaló anteriormente, esto tendría serias implicaciones sobre la libertad de los ciudadanos y equivaldría a la negación del conflicto. En Reyes Mate, como señala Barreto González (2010), la tolerancia deconstruye el concepto de totalidad como horizonte de la verdad, es decir, la pretensión de encontrar una verdad absoluta que permita comprender la realidad en su totalidad.

En oposición a visiones totalizantes de la verdad que puedan desencadenar conflictos, Reyes Mate fundamenta la tolerancia como virtud de la ciudadanía y de la construcción de paz en tres pilares: la búsqueda de verdad, la aceptación de la verdad por parte de los otros y el principio de humanidad; todos ellos relacionados entre sí con un propósito general: convivir pacíficamente a partir del reconocimiento de un elemento común en todas las sociedades, la dignidad humana. Sin embargo, cabe destacar que, hasta ahora, la dignidad humana no ha sido una realización histórica, sino un ideal por alcanzar. De ahí que sea necesario repensar la dignidad humana a partir de la víctima degradada (Mate, 2008b), esto es, quien padece las diferentes formas de violencia.

La búsqueda de la verdad hace referencia a la apertura individual y colectiva para reconocer el equívoco; la tolerancia requiere que los ciudadanos aprendan a escuchar y aceptar los hechos y razones que, aunque contrarios a las ideas propias, son verdaderos; no hacerlo es caer en el fanatismo que conduce a la violencia. Para Reyes Mate, la verdad, más que una posesión, es una búsqueda. Es decir, la verdad se busca, no se impone. En esa línea argumentativa, se puede retomar el principio kantiano de uso público de la razón por medio del cual la exposición de una máxima política en la sociedad tiene como propósito ser sometida a los cuestionamientos de la razón, para eliminar la desconfianza y posibilitar un acercamiento a la verdad (Kant, 2016). En otras palabras, aquello que es verdadero puede ser buscado y comprendido por todos a partir de los argumentos: "lo propio del ser humano es buscar la verdad y no regodearse con su posesión" (Mate, 2016, p. 68). Por otra parte, el principio de humanidad busca que ciudadanos y sociedades comprendan que 
las diferencias, aunque fuertes, no son profundas; de hecho, para Reyes Mate las diferencias pueden ser consideradas superficiales, toda vez que todos los ciudadanos poseen una dignidad humana común; aquello que genera conflictos no son más que diferencias de casa, es decir, producto de los azares del nacimiento, tales como la religión, la raza o la lengua.

Frente a las virtudes de la ciudadanía expresadas por Reyes Mate, llama la atención las similitudes con los valores requeridos para la paz que profesó la pedagogía alemana a finales del siglo XX. Esta consideró que "la tolerancia, la ayuda al prójimo y la conciencia de responsabilidad son los rasgos constitutivos de la educación para la paz de la gran unidad de vida" (Röhrs, 1992, p. 24). Esta convergencia permite avizorar la importancia de pensar la formación ciudadana desde dichas virtudes como un elemento de la construcción de paz.

\section{Sin justicia no hay paz}

Si la indiferencia es una forma de violencia, como se ha argumentado, y si la construcción de paz se relaciona con una formación ciudadana que se sustente en virtudes como la responsabilidad y la tolerancia, la memoria no solo es el punto de convergencia, sino también la posibilidad de hacer justicia a la violencia pasada, una forma de evitar que esta se repita. La memoria no solo trae al presente las injusticias que han tenido lugar, también devela las condiciones que las permitieron y posibilita la reflexión sobre la necesidad de emprender los cambios que las superen.

En ese orden de ideas, se habla de paz porque se reconoce que en la sociedad existe la violencia (Jiménez Bautista, 2012), al igual que se habla de justicia porque identificamos injusticias. En Reyes Mate, la idea de justicia puede concebirse como respuesta a actos que las sociedades consideran injustos, normalmente producto de un proceso de reflexión que permite develar el sometimiento o las condiciones que propician o legitiman la violencia y la injusticia que esta genera sobre las víctimas. 
Una paz pensada sin alterar la relación entre vencedores y vencidos, como señala Reyes Mate, busca la legitimación de las condiciones que propician la violencia; y requiere de la indiferencia para seguir perpetuando la violencia que ejercen los vencedores sobre los vencidos, dado que "si el más débil quiere vivir en paz tiene que aceptar las condiciones del fuerte" (Mate, 2016, p. 81). En ese sentido, la construcción de paz dependerá de mantener el statu quo y, por lo mismo, de negar los procesos de transformación de la sociedad. Dicho en otras palabras, es una paz contrarevolucionaria, tradicionalista, que se apega a los valores establecidos en la sociedad. Esto no sería problemático si las condiciones que se pretenden cambiar no fueran precisamente las que engendran -la violencia directa, estructural, cultural y por indiferencia-.

La visión de paz conforme con la legitimación de la hegemonía se encuentra en consonancia con lo expresado por Berón 0spina (2014) y Mate (2018), para quienes la modernidad privilegia la mirada sobre los demás sentidos, por eso "prima la abstracción de la generalidad observada, sobre la singularidad del particular que se expresa a través de la voz" (Berón Ospina, 2014, p. 178). Es decir, no interesa lo que los vencidos tienen para decir, sino lo que los vencedores imponen desde su forma de ver: la violencia como medio legítimo y necesario para asegurar su dominio. Es así como la paz de los vencedores dista mucho de ser una paz soportada en la responsabilidad y la tolerancia; se trata de una paz que niega la libertad y aviva los conflictos sociales con la indiferencia que muestra frente a lo singular, en concreto, hacia los vencidos. La paz de los vencedores impone la negación de los vencidos y el olvido de sus luchas y sus deseos de realizar una verdadera libertad.

Pensar la justicia en relación a la memoria como una forma para aportar a la construcción de paz hace necesario escapar de visiones restringidas 0 esencialistas del presente. Acudir a la memoria como forma de justicia busca alterar la relación entre el universalismo del poder y el particularismo de los individuos, pues negar lo particular es la primera forma de injusticia y, por esto mismo, una razón del conflicto social que, de no resolverse, solo puede devenir como violencia. Es fundamental pensar la construcción de paz desde la conciliación de lo universal con lo particular, y aquí la memoria es fundamental, en principio, porque esta no se sustenta en el ver, sino en el escuchar. La 
memoria se relaciona con la recuperación de la narración y por eso abre los expedientes sellados por la historia. Como señala Berón Ospina (2014), "si el poder no escucha, mucho menos recurrirá al tacto 0 al olfato para tener una comprensión social del mundo" (p. 178), dejando a los vencidos en el lugar que siempre han estado y estableciendo una paz que no es la paz de todos, sino la paz de los vencedores.

Esta forma de concebir la paz, de acuerdo con Reyes Mate (2008a), producto de la ruptura entre la política y la ética, trae como secuela que:

El individuo concreto no es capaz de ver en qué medida la dedicación a sus asuntos procura el bien común. Su actividad puede producir el bienestar de unos y la miseria de otros; él nunca sabrá en qué medida porque, de entrada, no interesa relacionar racionalmente la actividad privada con el bien general (p.112)

Por esta razón, para este autor tiene más utilidad abordar la justicia desde la injusticia que tratar de conceptualizarla desde posturas esencialistas que poco aportan a la comprensión de la realidad, dado que cada grupo o individuo puede entender por justicia una cosa diferente, máxime cuando se percibe en juego los privilegios alcanzados.

Expuesto así, la indiferencia aparece una vez más como el producto de una razón instrumental que se constituye en violencia al desconocer y someter al olvido aquello que no se encuentra alineado con el objetivo de progreso, en este caso, sus víctimas. La razón instrumental, en tanto es individualista y se encuentra condicionada por los vencedores, participa de que el ciudadano se muestre más preocupado por obrar de acuerdo a juicios particulares sobre lo correcto y lo incorrecto, que en reflexionar y actuar de acuerdo con un orden ético superior que le permita reconocer el sufrimiento producido y arrastrado por el progreso. Autores como Taub ( 2008) y Mate (2018) plantean la necesidad de repensar este suceder en términos de la teología política. La acción moral se constituye a partir del reconocimiento de la vulnerabilidad propia y en tal sentido de la posibilidad de ser el vencido; en síntesis, de reconocerse alguien que puede padecer la injusticia. Para la teología política acudir a la memoria permite redimir el sufrimiento de los vencidos por el progreso, en 
tanto que reconoce "la experiencia de la injusticia como el punto partida de la justicia" (Mate, 2006, p. 128). Recuperando el pasado de los vencidos se reconoce la posibilidad de otro presente: presente ausente.

La justicia entonces solo puede ser lograda haciendo uso de la memoria a través de cuatro aspectos: primero la recuperación del pasado; la memoria pone en evidencia la relación entre vencidos y vencedores y con ello los conflictos existentes entre estos - a diferencia de la historia de los vencedores, que se fundamenta en la negación u ocultamiento de los aspectos negativos del progreso, la memoria genera una ruptura con el relato oficial-. En la miseria de su presente y en su sufrimiento, el trabajo con la memoria ofrece los motivos para reclamar el derecho a la felicidad de los vencidos (Mate, 2009). La recuperación del pasado mantiene vigentes las luchas y los sueños que los vencidos perseguían; de esta forma, rescata lo particular como una forma de cuestionar lo universal y homogéneo del presente.

Segundo, develar las injusticias del pasado. La memoria desenmascara las injusticias que han tenido y tienen lugar, dando lugar a un conocimiento que aporta a la construcción de paz, pues, "si hay un conocimiento que legitime la injusticia o que encubra la evidencia, habrá que ponerlo entre paréntesis y esforzarse por pensar a partir de esa situación injusta" (Mate, 2013, p. 132). En otras palabras, a partir de la memoria no solo se interroga el presente y las situaciones que engendran la violencia, sino que al hacerlo se genera un conocimiento que ofrece alternativas, dado que, al escuchar las voces de los vencidos, se accede a la otra razón - la de los vencidos-y con ello se puede advertir alternativas de transformación social para la justicia y la paz social.

El tercer aspecto, referido a la reparación, vincula la visibilidad de las víctimas de la violencia, por un lado, y la posibilidad de ofrecer justicia alumbrando otras formas de realidad, por el otro. Como señala Reyes Mate (2008a): 
Finalmente, el cuarto aspecto es concebido como interrogación del presente; asume este como una posibilidad entre tantas otras que pudieron materializarse. El presente es el resultado de una lucha que ha dejado a un lado a los vencidos, edificando el progreso en su sufrimiento. Por ello, interrogar el presente es asumir la responsabilidad con las víctimas de las injusticias y fomentar una sociedad en la cual la responsabilidad y la tolerancia con lo particular se expresa en el reconocimiento de su derecho a realizarse. De acuerdo con Reyes Mate (2016), "si queremos entender lo que significa la responsabilidad ciudadana ante la violencia en general, tenemos que levantar acta de los daños que causa" (p. 91).

Así pues, ha de tenerse en cuenta que, tal vez, la única forma de conocer, no solo los daños que causa la violencia, sino las condiciones que la propician y cómo estas fueron impuestas, implica el trabajo de la memoria que recupera los aprendizajes del pasado requeridos para la construcción de paz y la formación ciudadana. No hacerlo significa negar que en la historia ha reinado la injusticia y continuar indiferente no solo frente a la violencia, sino también frente a la necesidad de transformar las condiciones que la propician. Reitérese, sin justicia no hay paz.

\section{Conclusiones}

La indiferencia es una forma de violencia en tanto normaliza las formas como la violencia se reproduce. Así, el progreso presenta la indiferencia como racional y excluye cualquier dilema moral frente al sufrimiento del otro. Ello se fundamenta en el bien de lo general a expensas de lo particular, legitimando, por tanto, formas de violencia e injusticia. En ese sentido, el actuar indiferente es contrario a la noción de ciudadanía como medio para alcanzar la convivencia social, pues permite la reproducción de las condiciones que malogran el disfrute de una vida humanamente concebida para todos los miembros de la sociedad. 
La construcción de paz requiere un proceso de formación ciudadana, a través del cual los seres humanos asuman la responsabilidad histórica, tanto de su tiempo como del tiempo pasado, para forjar un futuro diferente. En palabras de Reyes Mate (2008a):

\footnotetext{
nosotros podemos romper nuestras cadenas recordando que la actualización de las esperanzas pasadas e insatisfechas puede, en primer lugar, alumbrar nuestra conciencia respecto a la existencia de nuestras propias cadenas y, luego, transmitirnos su esperanza en virtud de la cual poder luchar contra las cadenas. La esperanza no nace de seres satisfechos sino insatisfechos (p. 207)
}

De ahí que sea importante partir de la memoria como una forma de superar la indiferencia y romper el continuum de la historia de violencia, que se ha constituido a partir de la injusticia sobre los vencidos y la complicidad de quienes se han negado a transformar las condiciones materiales que generan y legitiman la violencia.

\section{Conflicto de interés}

Los autores declaran la inexistencia de conflicto de interés con institución 0 asociación de cualquier índole.

\section{Referencias}

Adorno, T. (1998). Educación para la emancipación: conferencias y conversaciones con Hellmut Becker (1959-1969). Ediciones Morata.

Arteta, A. (2010). El mal público y su espectador. Anthropos, (228), 170-184.

Barreto González, D. (2010). Reyes Mate y el «nuevo pensamiento». Anthropos, (228), 150-159.

Benjamin, W. (2010). Pensamiento sobre el 'festival' de Gerhart Hauptmann. En Obras. Libro II/vol. 1 (pp. 57-61). Abada. 
Benjamin, W. (2012). Sobre el concepto de historia. En Obras (Vol. 2/Libro I, pp. 303-318). Abada.

Benjamin, W. (2020). Para una crítica de la violencia. En A. Hincapié-García \& E. Taub (Eds.), \& P. Oyarzún-Robles (Trad.), De Benjamin a Marcuse. Lecturas en torno a Para una Crítica de la violencia de Walter Benjamin (pp. 19-43).

Berón 0spina, A. A. (2014). El historiador como pensador: violencia y memoria. El caso colombiano (Tesis doctoral, Universidad Pablo de Olavide). Rio. http://hdl.handle.net/10433/1536

Cohen, H. (2004). El prójimo. Anthropos.

Fullat, 0. (1987). Filosofía de la educación: concepto y límites. Educar, (11), 5-15. https://www.raco.cat/index.php/Educar/article/view/42178

Galtung, J. (1995). La investigación sobre la paz y el conflicto en tiempos del cólera: Diez puntos para los futuros estudios de paz. Sociológica, (28), 235-250. http://www.sociologicamexico.azc.uam.mx/index.php/Sociologica/article/view/676

Galtung, J. (2003). Paz por medios pacíficos. Paz y conflicto, desarrollo y civilización. Bakeaz.

Goffman, E. (2006). Estigma. La identidad deteriorada. Amorrortu

Hegel, G. W. F. (2004). Principios de la filosofía del derecho. Sudamericana.

Hincapié García, A. (2016a). Formación y praxis pedagógica revolucionaria: los escritos de juventud de Marx y Benjamin. Andamios, 13(32), 257-279. https://andamios.uacm.edu.mx/index.php/andamios/article/view/532/880

Hincapié García, A. (2016b). 'Imaginación política', historia e impulso anti-narrativo. El retorno revolucionario de Walter Benjamin (1892-1940). En Modernidad y política. Sobre la pregunta antropológica (pp. 43-70). Ediciones UNAULA. 
Jiménez Bautista, F. (2012). Conocer para comprender la violencia: origen, causas y realidad. Convergencia, Revista de Ciencias Sociales, 19(58), 13-52. https://doi.org/10.29101/crcs.v0i58.1091

Kant, I. (2016). La paz perpetua (2 ${ }^{\mathrm{a}}$ ed.). Alianza Editorial.

Köhle, K. (1974). Contenidos y métodos de la pedagogía de la paz. Educación, (10), 89-97.

Mate, M. R. (1998). Heidegger y el judaísmo: y sobre La tolerancia compasiva. Anthropos.

Mate, M. R. (2003). Memoria de Auschwitz. Actualidad moral y política. Trotta.

Mate, M. R. (2006). Contra lo políticamente correcto. Política, memoria y justicia. Altamira.

Mate, M. R. (2008a). La razón de los vencidos ( $2^{\mathrm{a}}$ ed.). Anthropos.

Mate, M. R. (2008b). Primo Levi, el testigo. Una semblanza en el XX aniversario de su desaparición. En E. Madina, M. R. Mate, M. Rubio, \& J. A. Zamora (Eds.), El perdón, virtud política. En torno a Primo Levi (pp. 11-31). Anthropos.

Mate, M. R. (2009). Medianoche en la historia ( $2^{\mathrm{a}} \mathrm{ed}$.). Trotta.

Mate, M. R. (2011). Tratado de la injusticia. Anthropos.

Mate, M. R. (2013). La piedra desechada. Trotta.

Mate, M. R. (2016). Ciudadanos y no súbditos. Guía en la ciudad democrática. Ediciones USTA.

Mate, M. R. (2018). El tiempo, tribunal de la historia. Trotta.

Piñeres Sus, J. D. (2016). Antropología e idealidad. Algunas reflexiones sobre la crueldad y superfluidad. En B. Escobar-Garcia (Ed.), Modernidad y política. Sobre la pregunta antropológica (pp. 19-42). Ediciones UNAULA. 
Piñeres Sus, J. D. (2017). Lo humano como ideal regulativo: imaginación antropológica: cultura, formación y antropología negativa. Fondo Editorial FCSH.

Röhrs, H. (1992). El afianzamiento de una paz mundial permanente por medio de la educación para la paz. Educación, (46), 17-29.

Taub, E. (2008). La modernidad atravesada. Teología política y Mesianismo. Miño y Davila.

Zdarzil, H. (1978). Educación para la paz. Educación, (18), 110-120. 